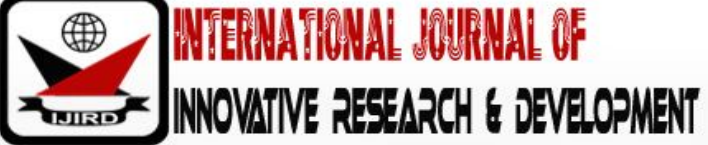

ISSN 2278 - 0211 (Online)

\section{Predicting Market Value of Egyptian Premier League Players}

Dina Akmal
Student, M.Sc. , Department of Operations Research and Decision Support, Cairo University, Egypt
Mohamed Saleh
Professor, Department of Operations Research and Decision Support, Cairo University, Egypt
Ehab El-Khodary
Nedaa Agami
Associate Professor, Department of Operations Research and Decision Support, Cairo University, Egypt
Lecturer, Department of Operations Research and Decision Support, Cairo University, Egypt

\begin{abstract}
:
Prediction of players' market value for the most popular sport on the planet through a data driven approach has no more became an option. In the last decade, football transformed from a sport to an industry with football players as the most valuable asset. Despite this fact, there hasn't been a systematic framework to address simple questions such: What really determines the value of a footballer? How much should a club pay when purchasing a certain player in a certain position on pitch in a certain season? Based on which criteria such that any subjectivity is eliminated? With the right answers to these questions, in the right time, a club as a revenue maker is assured to gain an unparalleled edge to its rivals. The Egyptian Premier League (EPL) is one of the top 5 leagues across Africa with total net worth of 105, 900 million Euros and still lacks a scientific approach to assign a proper estimate for a player's market value in a given season. In an attempt to bridge this gap, this research presents an end-to-end fully data driven scientific framework covering all given functions and hence positions of a footballer on pitch, be it a defender, midfielder, attacker or a goalkeeper. Players are first segmented as either: elite, highpotential or mainstream, according to their history of performance and then a prediction model is applied to predict the expected future market value accordingly. The results were tested and validated on real data and the model evaluation showed very promising results.
\end{abstract}

Keywords: Egyptian Premier League (EPL), football, market value, segmentation, prediction

\section{Introduction}

Sports data has recently been a hot topic and a fertile area of research for both statisticians and researchers. Football, known to be the most popular sport on this planet, failed to appear in most of the statistical studies due to the difficulty to collect and organize data on this sport. Further to that, it has been challenging to assess the singular impact, and hence value, of an individual player in a team sport with the nature of football. The game now has become big business, with huge sums of money in motion especially on the transfer of players, with many stakeholders interconnected worldwide.

The transformation of football from a sport to an industry, and further to a business, came from the clubs attitude and vision for themselves that they are no more exclusively venues for sport and entertainment, but also revenue generators and profit makers whenever possible. It all came from the clubs' need to cover costs and consequently their desire to acquire not only technically leveraged players but also "fan appealing" players to ensure their fans contentment which pushes the price cap of players' price to hit dynamic ceilings.

Egypt has exported to the world many excellent examples of professional football players with market values that always called controversy like the deal sealed by Chelsea FC for a striking 11 million British pounds, which made it at the time the most expensive transfer deal involving an Egyptian player. Ahmed Hossam (Mido), who was traded from the Netherlands Ajax FC to France Marseille FC for 13 million Euros, previously held that title. And most recently Mohamed Salah (Mo Salah) who was transferred from Italy Roma FC to the English Liverpool FC for 31.5 million Euros and currently is worth 150 million.

On the other hand, Egypt has also imported to its' leagues players and coaches and placed huge sums of money for these purchases to take place where some have proven their success by placing clubs in better positions and acquiring more revenues while others did not. Examples of these purchases are Ahly's protégées coach Manuel Jose who was paid US \$70,000 per month, Zamalek's Ghanian's attacker Agogo for 1.2 million Euros, former Egyptian national team's coach Marco Tardelli who was paid a monthly salary of US $\$ 40,000$ and Bob Bradley who was paid US $\$ 37,000$ per month.

With huge sums of money consistently in motion, essential questions must be clearly answered such as: What are the key determinants of a player's price? How much should a club pay when purchasing a certain player in a certain position on pitch in a certain season? There has not been a systematic scientific framework, and hence criteria, to address these 
questions or explore its distinctive features as no football player have exactly similar characteristics to another. Therefore, different clubs will get different financial value for the same player.

The objective of this research is to find the relationship between market value of players and their history of performance on pitch in previous seasons. In this paper, a consolidated fully data driven framework is developed for this purpose. At first, a segmentation model is applied to segment players according to their on-pitch performance into 3 classes: elite, high potential and mainstream. Then a prediction model is used to predict the closest estimate to their real market value by segment depending on their position on pitch. A fair market would assign a higher value to a player with high performance. After obtaining a player's market value and his performance through the right indicators, the relation between the 2 is studied and uncovered.

The remaining sections of this paper are organized as follows: first the problem addressed is formalized briefly then the previous researches addressing the same problem in other premier leagues are demonstrated. Following that, the proposed conceptual framework for the research problem is illustrated and explained in details. Afterwards, the champion models as well as the generated results are discussed. Finally, the conclusion and possible future work are highlighted.

\section{Problem Definition}

Football players' transfer now in Egypt is playing a more important role than ever as the league continues almost without audience after it had been called off at the halfway stage in 2012 following the Port Said disaster, which left over 70 Ahly fans dead, being resumed the following year. However, it was again cancelled just before the final four-team playoff after the army's ouster of its former President which led to a wave of unrest in the Egyptian football.

Playing behind closed doors meant no ticket sales or any sort of derived revenues from fans attending on match days. This even threw extra burden on other revenue generating channels such as players transfer and increased the necessity of a proper valuation mechanism for players. Not only that, it further calls for a deeper understanding of the market cost drivers and values to keep clubs' existing revenue streams and even find new ones.

All the previous calls for an essential need of clubs to have a reliable consistent approach for players' valuation in any given season to guarantee sealing the right deals and hence, increasing the generated revenues from such a main stream revenue.

\section{Previous Related Work}

All previous efforts that were ever dedicated in the sports analytics field to address the topic of players 'market valuation was done in European Leagues. No previous research was ever published targeting the EPL or any other league in the MENA region, which makes this research the first of its kind in terms of the market under study. However, several researches were presented on the topic for other European National Leagues such as: the English Premier League, German Premier League (Bundesliga), Spanish League (La Liga) and the Italian Premier League.

Previous researches in the field could generally be split into 2 types. One type attempted to answer questions related to the key determinants and significant factors when making a transfer or a player's purchase decision while the other focused on predictive modelling efforts to forecast the expected market value of a player in a given season. In that prospect, the main researches tackling both types will be briefly discussed in the rest of this section.

In an attempt to identify the most influential factors in setting a player's equivalent market value, Eschweiler and Vieth (2004) questioned to what extend do club-specific or player-specific factors influence transfer prices in the German soccer league. It was found that for seasons 1997/ 1998 throughout 2002/ 2003 the extent of transfer price is significantly dependent on club and player specific factors. Club-specific factors were found to be: revenues from main sponsor, average number of spectators from prior season, and qualification for international competition in next season, where as age and tenure (number of games played) were proven to be the most significant player-specific factors. For the same league and the same research question, Huebl and Swieter (2002) concluded a concave relationship between age as well as number of games and salary. They also discovered a positive relationship between variables such as player in national team (yes/no), origin, and player position (goalkeeper, defender and midfielder).Similarly, other researches were done for the Spanish Premier League. For instance, Garcia-del-Barrio and Pujol (2004) on their quest for discovery whether there is a winner-take-all effect concluded that index of performance, reputation, superstar, age, international appearance, games in the Champions League/ UEFA, and position caused the existence of 2 segments. The winner-take-all effect leads to strong bargaining power of superstars.

The vast majority of studies conducted in this topic were for the English Premier League where researchers found that the largest costs for teams were wages spent on players and transfer fees paid to acquire such players (Battle et. al., 2011).Carmichael, Forrest and Simmons (1999) used English Premier League data to model transfers using an Ordinary Least Squares (OLS) cross-sectional model. In their analysis, they found that age, games played in previous season, and goals scored in previous season were significant in determining transfer fees. Most of the scientific papers, including the ones highlighted earlier, put too much focus and weight from the analysis on number of goals scored by a player which gives a very clear bias towards players in attacking positions in the field. This makes such assessments unfair for players in non-attacking positions such as defenders, midfielders and undoubtedly goal keepers.

Finally, for the Italian Premier League, Lucifora and Simmons (2003) investigated what shaped players' earnings function in the league and if there was a superstar affect. They found that variables representing player experience including age, appearances, performance (described through number of goals, number of strikes, and number of assists) in addition to position and reputation were the most significant player related variables yielding a convex structure across some performance measures.

This is upon discussing significant variables determined from previous research efforts in each league. As for the 
topic of predicting the market value of players, there have been several attempts -- all in European leagues -- to address the issue as discussed in the remaining parts of this section.

Radu Tunaru, Ephraim Clark, Howard Viney (2014) tried to uncover the constituents of the equation of a footballer's price through a mathematical stochastic model based on the geometric Brownian motion, Poisson processes and jump-diffusion process. They concluded that Player value varies from club to club, depending on club turnover and the total number of performance points generated by the entire team. Despite the many advantages of the model, a problem of multidimensionality was faced and hence, the problem was classified of a computational nature.

On another hand, Erik van den Berg (2011) in their research questioned the valuation of football players in the English Premier League. They also analyzed the variance in transfer fees paid and received by English Premier League clubs in 2 consecutive seasons. In addition to that, they investigated whether asset characteristics are the main determinants of value of player (such as buying and selling club characteristics) through a Simple Linear Regression Model. Their work reached a conclusion that individual player performance and intrinsic ability are prominent determinants of transfer fees. As for the second point of investigation, they concluded that the buying club's size brings transfer fees up to levels unexplained by measures of performance or ability which lends credence to the use of a bargaining framework. They clearly stated that many prominent variables influencing transfer prices were not available in their research such as remaining contract duration of transferred players which is classified as a critical determinant of bargaining power in transfer negotiations. Furthermore, they emphasized that the current measures understate the teamelement of the game and fail to adequately distinguish between the various player actions that are relevant to the outcome of games, therefore fail to measure a player's actual contribution as his influence on the probability of the team to win or lose a game.

In the same prospect, Yuan He (insert year) aimed to predict market value of top players using statistical modeling techniques. Four modeling techniques were used in his research: OLS, K-Nearest Neighbors (with different $\mathrm{k}$ values), Ridge Regression (with different lambda values) and Principal Component Regression (PCR) (with different $\mathrm{k}$ values). A cross validation of 10 fold has been used for each of the techniques, which meant that the model was trained on most of the data matrix and was tested on one fold. According to his research, the best model was PCR with k=15. On the limitations of the research front, it was stated that used predictors did not cover every possible factor that could affect players' market value. Players from different positions should have different criteria for judging their performance. In addition to these missing variables from the research, it was also highlighted that other crucial on pitch related variables such as number of assists, number of clean sheets, tackles per game, would have made it much better for the evaluation of midfielders (assists), defenders (tackles) and goalkeepers (clean sheets).

Jeroen Ruijg, and Hans van Ophem (2014) used a linear regression model. Upon concluding their research, it was stated that significance was very modest, where only age and the number of minutes played in the season were found to have an effect before the transfer. Age was found to have a negative effect on the transfer fee while playing games was found to have a positive effect. They also highlighted that taller players seem to be less attractive on the player market. In addition to that, it was declared that playing and not being a substitute usually increases the transfer fee.However, a negative effect of red cards received was revealed. Other generated insights were clarified such as being left-footed is not a positive player characteristic. Moreover, attackers, midfielders and defenders were found to be in higher demand than goalkeepers. A final revealed surprising result was that the number of goals scored did not seem to have a big impact on the player's market value.

Cornelius Arndt and Ulf Brefeld (2017) performed a multi-task regression-based approach for predicting future performances of football players. The multi-task approach was said to allow researchers to simultaneously learn individual player models as offsets to a general model. Multi-task variants of Ridge regression and $\varepsilon$-Support Vector Regression (SVR) were devised, with a hashed joint feature space. Relevant, i.e. significant, features for the prediction were identified by a modified recursive feature elimination strategy. Upon concluding the generated results, it was stated that the proposed multi- task generalizations of Ridge regression and SVR allowed efficiently learning player-specific models. Empirical results on real-world data from the German Bundesliga showed that data was often too sparse for learning individual player models. The single-task models thus often performed better than the multitask extensions.

Finally, Miao He, Ricardo Cachucho, and Arno Knobbe (year not mentioned) attempted to investigate how the market value and performance of players in the Spanish Premier League could be modeled by using public data sources through applying a regression model to predict the real market value and assess a player's performance. The model applied only to forward players. However, they recommended its extension to other positions with a specifically league tailored model. They further recommended extending the study up to include all other European leagues. Yet, they made it clear that even if this recommendation was put to real action, it would still be a problem to deal with players who have never been valued before (never been transferred) nor their values were voted for by voting system. 


\section{Proposed Conceptual Framework}

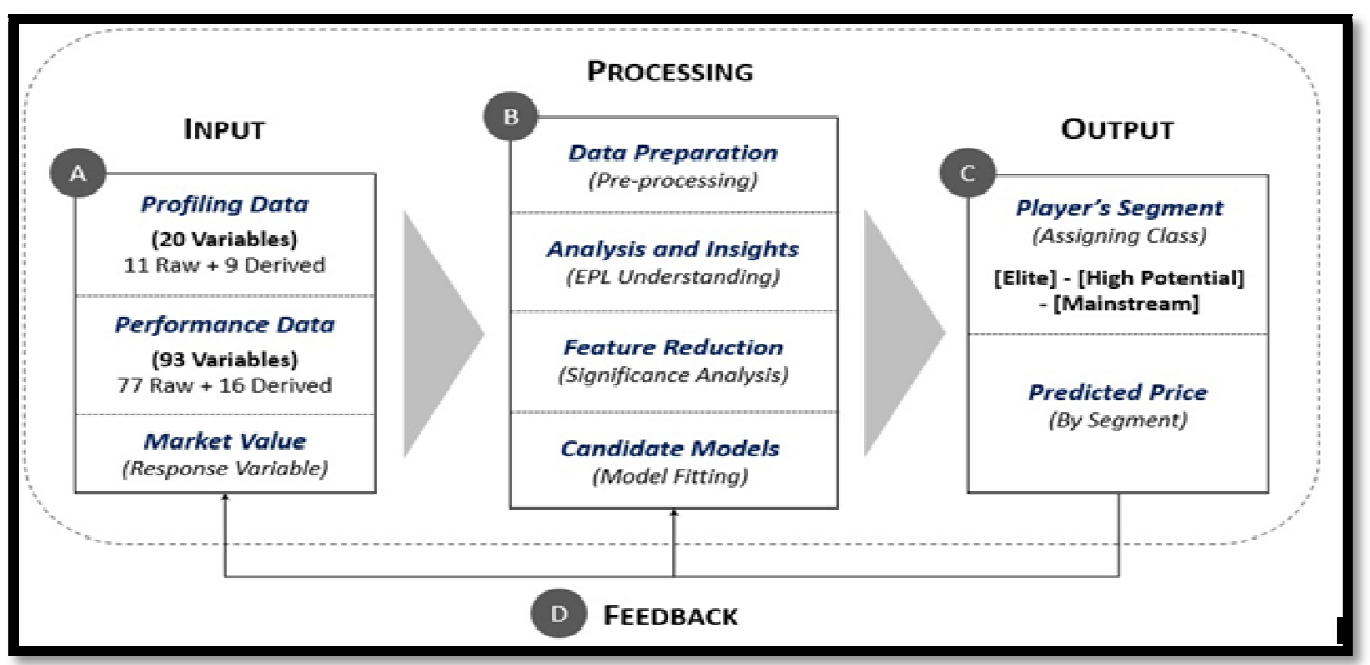

Figure 1: System Structure Diagram of the Proposed Framework

Figure 1 demonstrates the conceptual framework of the proposed solution represented by a System Structure Diagram (SSD) split into 4 key components as follows: Inputs, Processing, Output and Feedback, each of which shall be explained in details below.

\subsection{Inputs}

In this study, data was collected from 2 main data sources: KoraStats ${ }^{1}$ and Transfer Markt ${ }^{2}$, for 3 consecutive seasons (2015-2016, 2016-2017, and 2017-2018). The former captures data about player's on pitch performance during his appearances in a given season represented in 90 variables (either profiling or performance data elements) listed and summarized in appendix 1. They cover all player's functions in the match according to their position on pitch as shown in Figure 2.Functions and how they are linked to positions are explained in table 1.

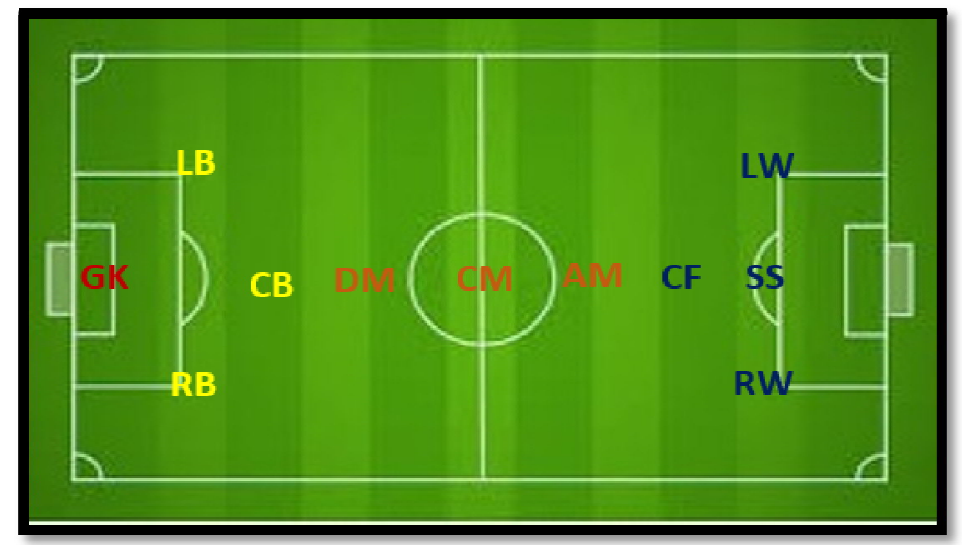

Figure 2: Distribution of Players' Position on Pitch

\begin{tabular}{|c|c|c|}
\hline Symbol & Position Name & Function on Pitch \\
\hline GK & Goalkeeper & Goalkeeper \\
\hline LB & Left Back & \multirow{2}{*}{ Defense } \\
\hline RB & Right Back & \\
\hline CB & Center Back & \multirow{2}{*}{ Midfield } \\
\hline DM & Defending Midfield & \multirow{2}{*}{ Attacking } \\
\hline CM & Center Midfield & \multirow{2}{*}{} \\
\hline AM & Attacking Midfield & \\
\hline CF & Center Front & \\
\hline LW & Left Wing & \\
\hline SS & Second Striker & \\
\hline LW & Left Wing \\
\hline
\end{tabular}

${ }^{1}$ KoraStats is a local Egyptian company owned by EgyNile. It is specialized in collecting live data about players' performance every match for the EPL each season. (https:/ / korastats.com/ en/)

2 TransferMarkt is a German-based website owned by Axel Springer.It has footballing information such as scores, results, statistics, transfer news and others. (https:/ / www.transfermarkt.co.uk/ ) 
In addition to the previous set of variables, the market value (price) data of players was extracted from Transfer Markt. It was used later in the modelling stage as the response variable.

\subsection{Processing}

The processing phase was designed and implemented in 4 consecutive stages: Data Preparation, Analysis and Insights, Feature Reduction and Candidate Models. For stage 1, raw data was provided from the primary data source Kora Stats, and merged with the second by player's name, position on pitch, and number in the team as the joining links to ensure accuracy of the merge process.

In order to handle the missing values in the data fields, several data imputation techniques were appliedsuch as mean imputation and regression imputation on the quantitative variables as relevant. In some occurrences where imputation would not be applicable (basically market information variables)such as contract until or basic player's information such as height, the entire player record was excluded from the analysis

Additional derived variables were calculated from raw data as mentioned in appendix 1.Derived variables were calculated for the purpose of obtaining more indicative representation and meaningful insights about a players' performance. An example of a derived variable is contribution in season which is calculated as the summation of matches played as sub and matches played as line undivided by the total number of matches in the league. Worth mentioning that the championship consists of 18 competing teams in a "home" and "away" league system to reach a total of 34 games where the teams compete against each other, once on their home ground and another on the other's team ground. Accordingly, the newly created contribution in season variable is a percentage that provides an estimate of a given player's involvement (in terms of percentage of appearance) with his team in the entire league in a given season.

Another example of a derived variable is the player engagement index. It is calculated as the summation of all variables that help describe the behavior of a player on pitch, both negatively and positively, and reflect the overall level of player engagement during a match. Examples of variables indicating negative behavior are fouls awarded, penalty awarded, yellow card, second yellow card, red card and off-sides whereas fouls committed and penalty committed are indicators of positive on pitch behavior. The player's attitude in a given match when engaged with other players of opposing team holds both possibilities for being awarded or committing fouls and penalties as well as receiving disciplinary cards, either yellow or red. The purpose of this variable was to represent from a behavioral assessment perspective the level of a player's engagement in matches during a given season. Derived variables were calculated to gain insights about a player's performance from raw on pitch data provided as well as unfold their significance when analyzed against the market value of the player.

The second stage of the processing phase was generating insights about the EPL and its players' prices against their performance on pitch to help focus on the most significant variables in the price setting equation. As mentioned in section3, previous researches in the field addressing other leagues stated some variables that proved to be significant in determining the market value of a player and affecting its variation. However, it was proven otherwise for the EPL. For example, player's preferred foot (right, left or both) was previously proven a significant factor when it comes to setting the player's equivalent market value in the sense that a player who has the capability of playing with both feet is more valued in terms of price than his rivals with the capability to only play with one.Yet according to the conducted analysis, this was not the case in the EPL.Another example is the player's age which when studied against market value was proven insignificant in the EPL despite proving otherwise in other leagues. Although it might even seem counterintuitive, many insights were very specific to the different nature of the EPL.

In the third stage of the processing phase, statistical significance of performance variables (either raw or derived) in relation to the market value of players was assessed. Feature reduction was done by implementing forward step-wise regression. Multi-collinearity of variables was examined and correlation matrices were created. Variables whose correlation coefficient had an absolute value greater than 0.75 were identified and only one of them was kept. This recursive elimination process ensured that no redundant information would be provided as input to the model, hence ensuring an efficient modelling process. An example of a correlation matrix for the goalkeeper model variables post feature reduction is illustrated in figure 3 . 


\begin{tabular}{|c|c|c|c|c|c|c|c|c|c|c|c|c|c|c|}
\hline Value in $\mathrm{EGP}$ - & 0.01 & 0.33 & 0.42 & 0.09 & 0.09 & 0.11 & 0.05 & 0.02 & -0.03 & -0.06 & 0.02 & 0.02 & 0.04 & 1 \\
\hline One on One Save Per : & 0.27 & 0.25 & -0.13 & -0.23 & -0.29 & 0.41 & -0.13 & 0.04 & 0.68 & 0.24 & -0.1 & 0.02 & 1 & 0.04 \\
\hline Long Pass Success Per- & 0.03 & -0.22 & -0.15 & 0.03 & 0.12 & 0.11 & 0.22 & 0.1 & 0.05 & 0.02 & 0.21 & 1 & 0.02 & 0.02 \\
\hline Ball Received Success - & -0.1 & -0.11 & 0.1 & -0.27 & 0.34 & 0.21 & 0.4 & 0.73 & 0.02 & 0.04 & 1 & 0.21 & -0.1 & 0.02 \\
\hline Penalty Save Per- & 0.27 & 0.11 & -0.12 & -0.13 & -0.19 & 0.24 & 0.01 & 0.18 & 0.52 & 1 & 0.04 & 0.02 & 0.24 & -0.06 \\
\hline Goal Conceded- & 0.28 & 0.18 & -0.2 & -0.34 & -0.26 & 0.51 & -0.01 & 0.24 & 1 & 0.52 & 0.02 & 0.05 & 0.68 & -0.03 \\
\hline Clear- & 0.07 & 0.04 & 0.1 & -0.27 & 0.15 & 0.24 & 0.29 & 1 & 0.24 & 0.18 & 0.73 & 0.1 & 0.04 & 0.02 \\
\hline Tackle Clear Per- & -0.11 & -0.23 & 0.06 & -0.13 & 0.41 & 0.25 & 1 & 0.29 & -0.01 & 0.01 & 0.4 & 0.22 & -0.13 & 0.05 \\
\hline Intercept Won Per- & 0.17 & 0.08 & -0.12 & -0.55 & -0.02 & 1 & 0.25 & 0.24 & 0.51 & 0.24 & 0.21 & 0.11 & 0.41 & 0.11 \\
\hline Aerial Won Per - & $=0.15$ & -0.12 & 0.07 & 0.05 & 1 & -0.02 & 0.41 & 0.15 & -0.26 & -0.19 & 0.34 & 0.12 & -0.29 & 0.09 \\
\hline Season_Num- & 0.1 & 0.07 & 0.06 & 1 & 0.05 & -0.55 & -0.13 & -0.27 & -0.34 & -0.13 & -0.27 & 0.03 & -0.23 & 0.09 \\
\hline Club Points - & -0.03 & 0.38 & 1 & 0.06 & 0.07 & -0.12 & 0.06 & 0.1 & -0.2 & -0.12 & 0.1 & -0.15 & -0.13 & 0.42 \\
\hline Height- & 0.27 & 1 & 0.38 & 0.07 & -0.12 & 0.08 & -0.23 & 0.04 & 0.18 & 0.11 & -0.11 & -0.22 & 025 & 0.33 \\
\hline Age - & 1 & 0.27 & -0.03 & 0.1 & -0.15 & 0.17 & -0.11 & 0.07 & 0.28 & 0.27 & -0.1 & 0.03 & 0.27 & 0.01 \\
\hline & $\&$ & 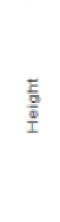 & 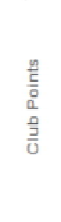 & 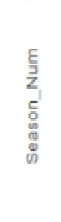 & 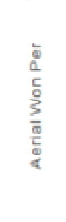 & 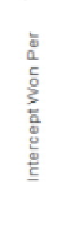 & 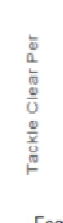 & 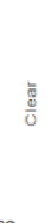 & 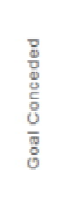 & 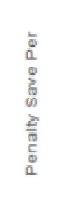 & 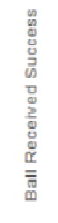 & 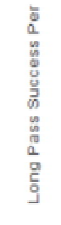 & 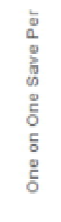 & 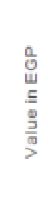 \\
\hline & & & & & & Corr & Fea & & & & & & & \\
\hline
\end{tabular}

Figure 3: Correlation Matrix For The Goalkeeper Model Variables Post Feature Reduction

Finally in the fourth and last stage, candidate models identified as potentially successful for tackling similar problems as reported in the contemporary literature are shortlisted for testing on the available EPL data. For the segmentation part, K-Means was on top of the list since the segmentation requirements were quite straightforward especially after aggregating the variables by relevance according to the players' function on pitch. Further details on the implementation steps will be provided in section 4.

As for the market value prediction potential models, several approaches were reported in previous studies to have yielded promising results. Regression models of various types were frequently applied and proven successful for the same problem in other European leagues. Best results in terms of explanatory power of the modelindicated by r-squared ${ }^{3}$ were mostly achieved by Lasso, followed by Ridge then OLS regression. PCR usually generated themodest of all results according to r-squared figures.KNN and ANN were competent in some cases when assessed according to the root mean squared error ${ }^{4}$ (RMSE).

\subsection{Output}

The output of the model was two-fold. First, a segmentation model was developed to classify players in a given function as belonging to one of 3 segments: elite, high potential or mainstream, according to his position and consequently function on pitch, where best performers are segmented as elite and worst performers as mainstream. A price bracket is tied to each of the 3segments.

After segmentation, the actual price is predicted through a statistical based model for each specific function of the 4 primary ones on pitch: goalkeeper, midfielder, defender and attacker. The main objective of this stage was to enhance the accuracy of market value prediction. This is achieved through tightening the price bracket for players when tiered by segment instead of the wider price range of all players on pitch collectively.

The second stage constitutes the main output where a statistical based prediction model is fitted by segment (elite, high potential and mainstream) using historical performance data of 3 consecutive seasons to predict the equivalent market value of a player in a given season.

A different model is developed for each of the 4 distinctive on pitch functions as recommended by the vast majority of previous researches in the same domain. The idea is to ensure assigning the right weights to the different skills needed according to the player's position since it is unfair to assess the technical capabilities of a defender by the number of goals scored or the attacker by the number of blocks. Likewise, a goalkeeper cannot be assessed by the number of successful attempts on target and the midfielder by the number of goals saved.

\section{Champion Models and Results}

To address the research problem, several prediction models were implemented by segment for everyon-pitch function. Each model was tested and assessed in terms of its capability to accurately and effectively predict the equivalent market value of players in a given season. In this section, the model identified as the champion for every function is highlighted and the results are demonstrated separately.

${ }^{3} \mathrm{R}$-squared represents the squared correlation between the observed known outcome values and the predicted values by the model. It is usually used as an indicator to the explanatory power of a model.

${ }^{4} \mathrm{RMSE}$ is a measure of the model prediction error. It corresponds to the average difference between the observed known values of the outcome and the predicted value by the model 
First and foremost, it is worth mentioning that experts in the Egyptian football market were interviewed and asked explicitly about the most influential factors when making a player's purchase decision for a club in any given season. They all agreed that the 7 main ones are:

- The player's medical status (according to their history)

- His injury record throughout their entire career in football

- Ability to develop technical capabilities on pitch across seasons

- Uniqueness of skills amongst other players in the club

- Attitude during matches played

- Demand on the same player from other clubs

- The market purchase power

As mentioned earlier, the final output was generated in 2 steps. At first for every function on pitch, players were segmented as either elite, high-potential or mainstream. The segmentation was fully data driven. Besides the market value, variables that were found to be significant for every function were included as factors in the segmentation process. The main idea is to simply derive distinctive segments from the available data in order to understand how players varied in performance on pitch in any given season. Therefore, K-Means clustering algorithm was a suitable approach given how it works and hence, was identified as the champion segmentation model for all functions. It is well known as one of the simplest unsupervised learning algorithms that solve basic, conventional clustering problems. The procedure follows an easy way to classify a given dataset through a certain number of clusters (in this case $\mathrm{k}=3$ ) fixed in advance. The main idea is to define $\mathrm{k}$ centroids, one for each cluster and iteratively measure the distance between them. Final clusters are generated when the inter-distance between the clusters is maximized and the intra-distance between points falling in the same cluster is minimized. Further details on how the algorithm work can be found in (MacQueen, 1967).Figures 4 to 7 show a visual representation of the final clusters as well as the K-Means algorithm results for the goalkeeper, attacker, defender and midfielder functions respectively.

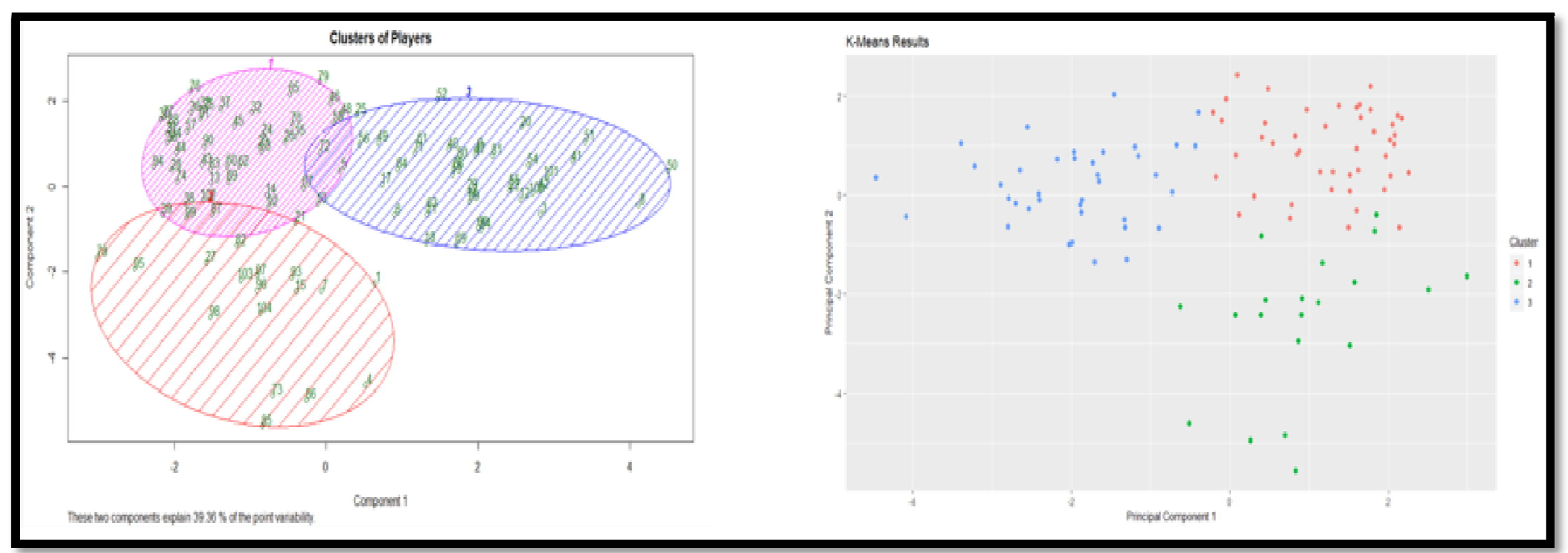

Figure 4: Final Clusters and K-Means Model Results for Goalkeeper Function

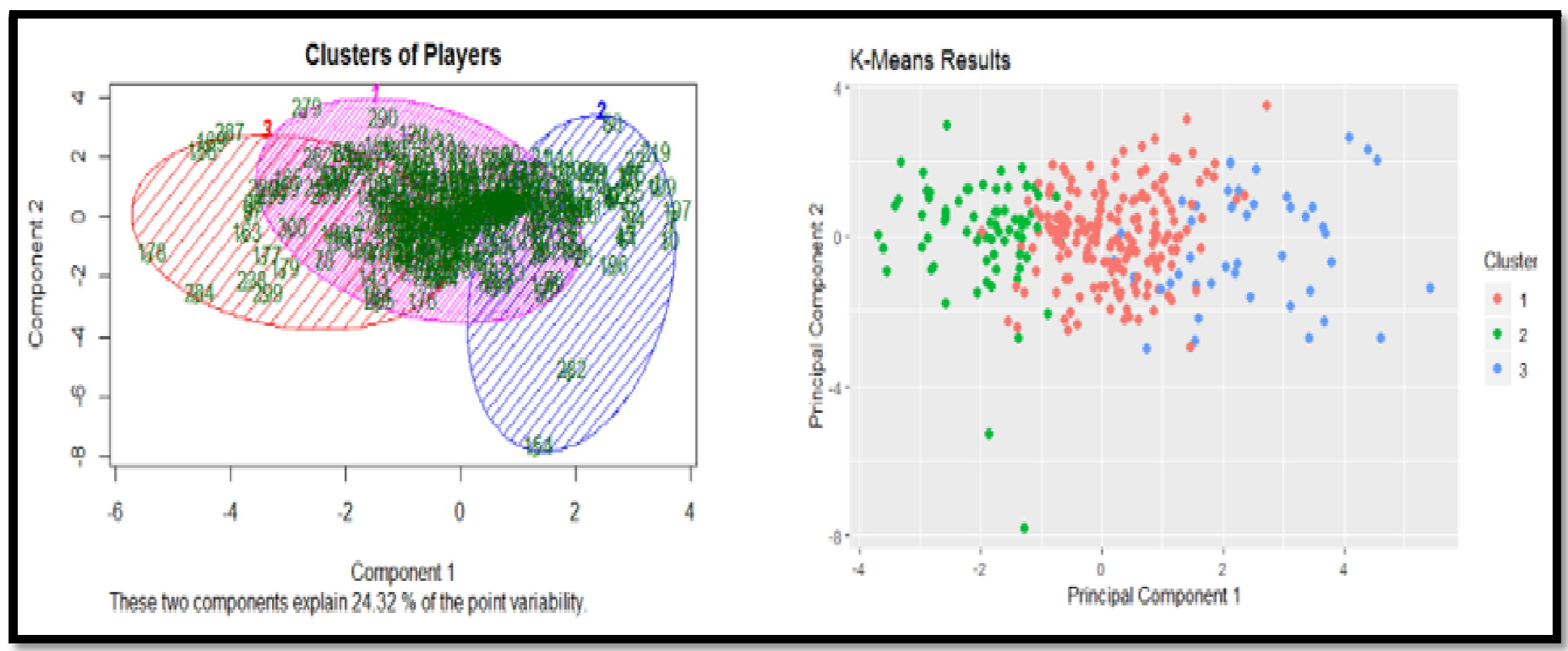

Figure 5: Final Clusters and K-Means Model Results for Attacker Function 


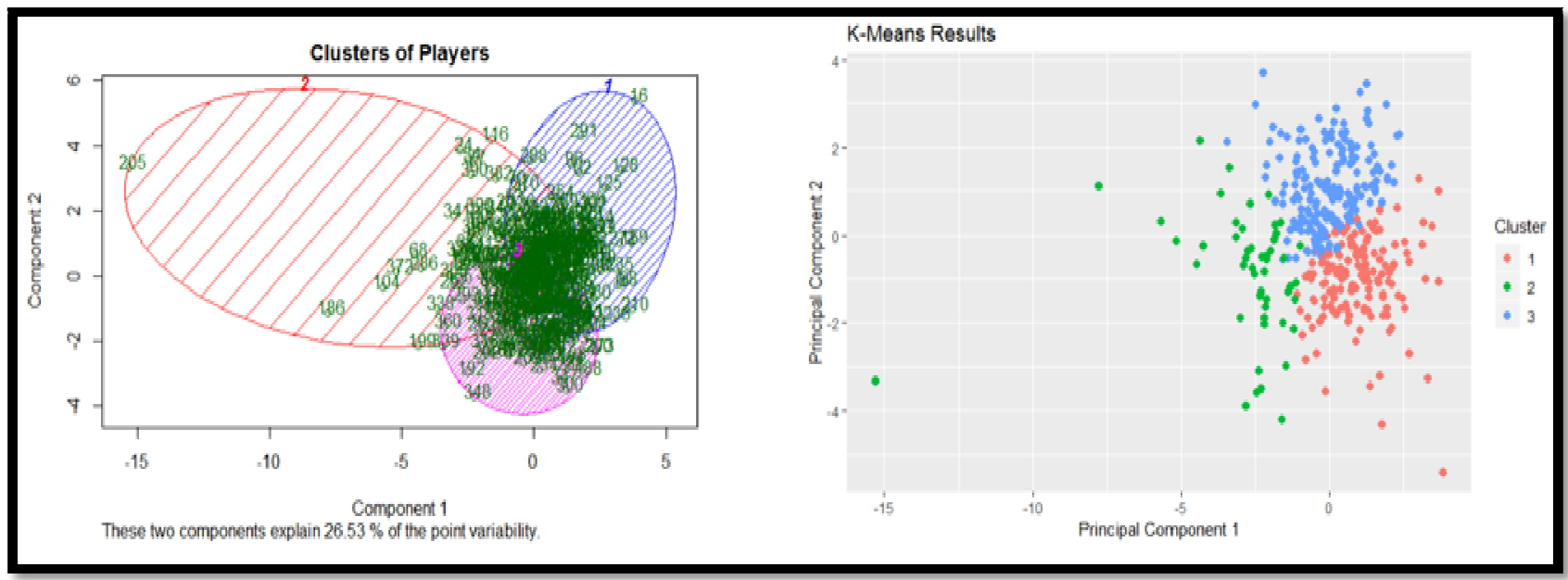

Figure 6: Final Clusters and K-Means Model Results for Defender Function

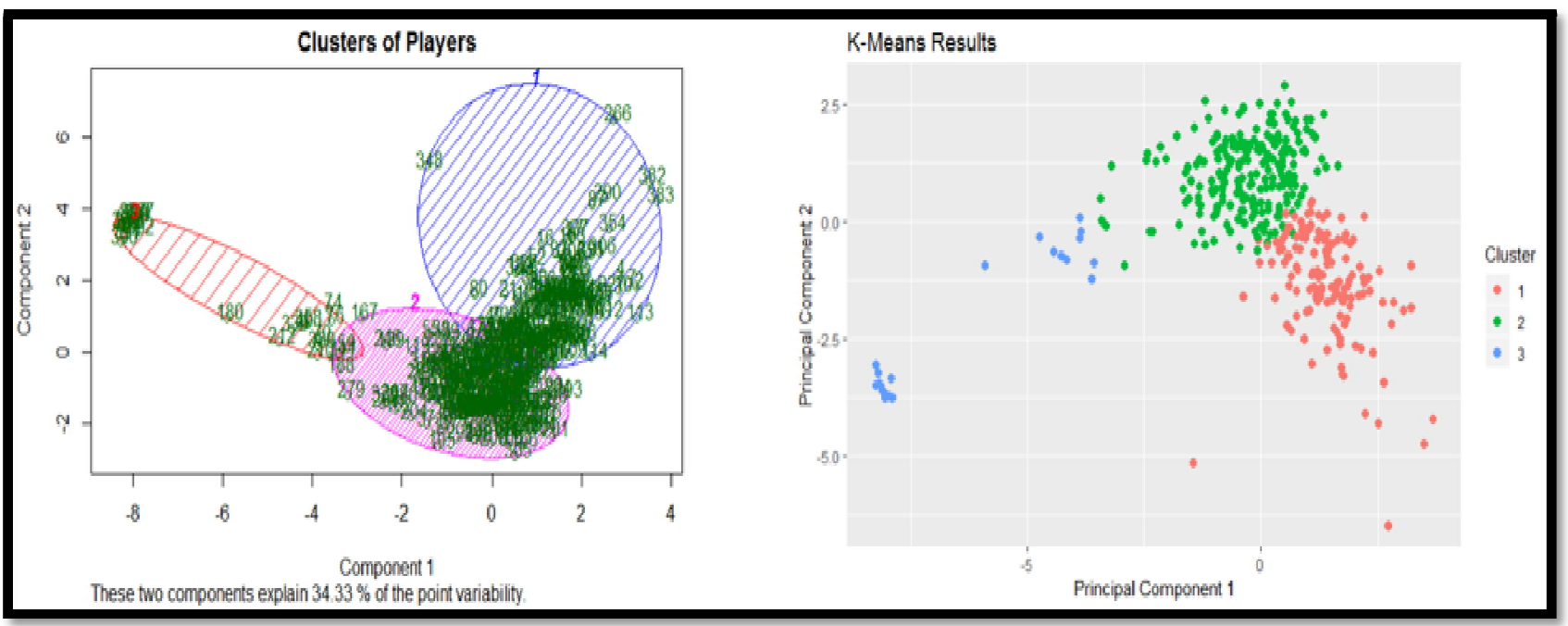

Figure 7: Final Clusters and K-Means Model Results for Midfielder Function

The rest of this section will discuss the champion prediction models and their results for the goalkeeper function followed by the attacker, then the defender and midfielder functions.

\subsection{Goalkeeper Function Models}

As reported in literature, regression models of various types have mostly proven successful and yielded good results when used to tackle the same research problem in other leagues. The most frequently applied ones were OLS, Ridge, Lasso and PCR. The last 3 are generally used when the standard linear model (OLS) performs poorly in a situation which mostly happens when there is a large multivariate dataset containing a number of variables superior to the number of samples.

The idea behind the Lasso model is to implement penalized regression which allows for creatingstill a linear regression model, but one that gets penalized for having too many variables included by adding a constraint in the equation (James et al. 2014, P. Bruce and Bruce 2017). This is also known as shrinkage or regularization methods.

To address the problem on hand, all the 4 linear regression models were applied by segment, tested for their effectiveness and compared to each other in terms of their explanatory power represented by r-squared. The differences in the RMSE were really minor and thus, r-squared was used to select the champion model. The Lasso model proved superior to the rest with a relatively decent gap. Results are summarized in table 2 .

\begin{tabular}{|c|c|c|c|}
\hline OLS & Ridge & Lasso & PCR \\
\hline $53 \%$ & $69 \%$ & $\mathbf{7 4 \%}$ & $21 \%$ \\
\hline \multicolumn{3}{|c|}{ Table 2: R-Squared Values for Linear } \\
Regression Model Attempts
\end{tabular}

To account for non-linearity, other attempts included ANN and KNN also for regression. The key differences lied in either the model structure (in terms of number of hidden nodes and number of hidden layers) for the former or the number of nearest neighbors $\mathrm{k}$ (training observations) that resulted in the minimum RMSE and hence generated the most accurate estimates of the response variable, i.e. market value.

For the ANN, different architectures and different activation functions, including logistic and tanh, were tested. However, the one hidden layer with one hidden node and a logistic activation function network resulted in the smallest 
RMSE on the test dataset. As for the KNN, different values for $\mathrm{k}$ where tested as shown in figure 8. The best RMSE was attained at $\mathrm{k}=7$.

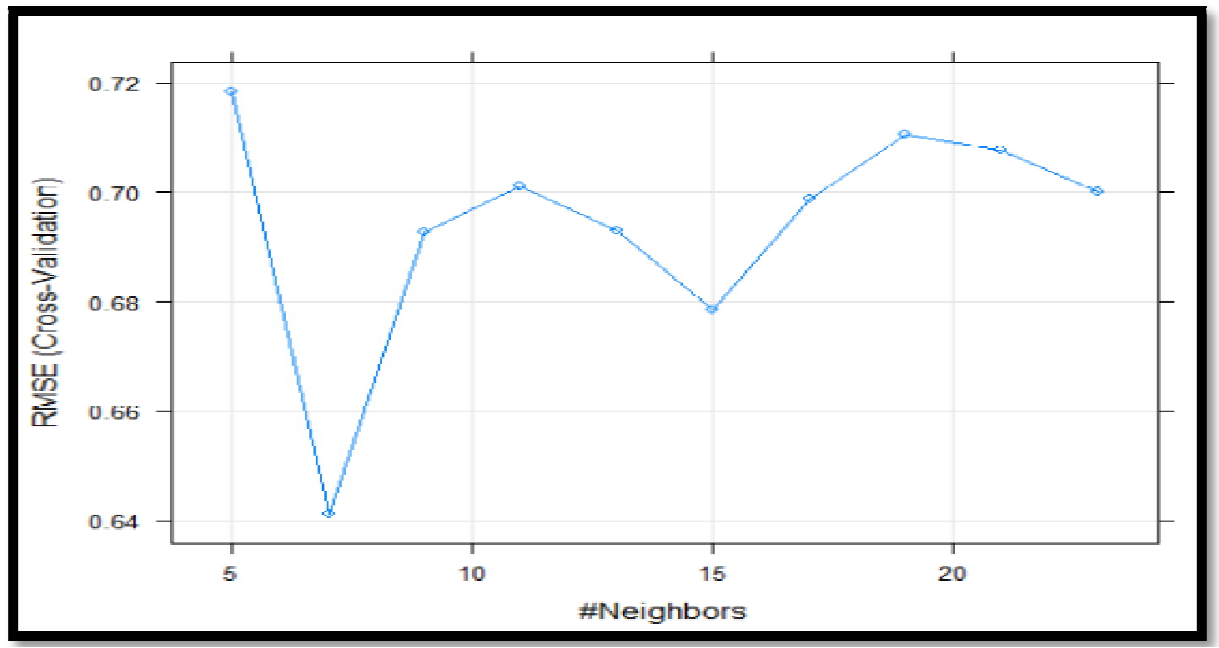

Figure 8: RMSE by Different K Values for the

Goalkeeper Function

It is worth mentioning that the KNN model was superior to the ANN in terms of RMSE. However, for the goalkeeper function, the Lasso instance proved superior to all. It had the best r-squared and RMSE values.

\subsection{Attacker Function Models}

Almost no previous study that addressed the same research problem attempted to build a separate prediction model for every function on pitch. Hence, there were no findings reported in literature for comparison purposes. Consequently, the market value prediction for the attacker, defender and midfielder functions was tested and assessed using the same set of models applied for that of the goalkeeper. In that sense, the 4 linear regression model types namely: OLS, Ridge, Lasso and PCR as well as the non-linear ones represented in ANN and KNN were tested and validated for their effectiveness for the attacker function by segment. However, the highest r-squared value for the linear models was achieved by OLS, followed by Lasso, then PCR and finally Ridge. This could be attributed to the nature and diversity of the on-pitch skills needed for players in attacking positions as well as the linear underlying relationship found between the on-pitch performance indicators for that specific function.

As for the non-linear model instances, the same architectures were examined for the ANN and the single hidden layer, single hidden node with a logistic activation function network was still in that case superior to the others. The KNN model was still better in terms of the resulting RMSE. However, the best value yielding the minimum RMSE was $\mathrm{k}=19$ for the attacker function. Figure 9 demonstrates the RSME achieved by different values for $\mathrm{k}$.

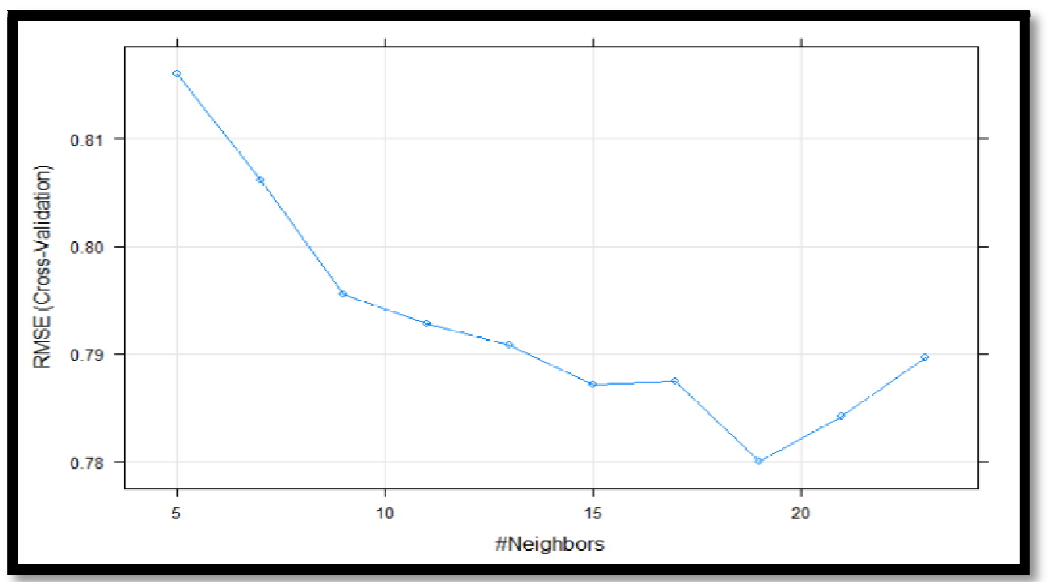

Figure 9: RMSE by Different K Values for the Attacker Function

Although the standard linear model OLS had the best explanatory power for the attacker function, Lasso still resulted in the minimum achieved RMSE across all models developed.

\subsection{Defender Function Models}

Similar to the goalkeeper and attacker functions, the same set of predictive models, both linear and non-linear, was applied and tested for that of the defender. In this case, the highest r-squared value achieved by a linear model was for Lasso, followed by OLS, Ridge and PCR where almost all the 3 resulted in approximately the same r-squared value. Because 
of the high similarity between performance variables indicating the skills possessed by players in the defender position, the Lasso model was proven to be the most effective due to the model nature and the penalization concept.

However, for the non-linear instances, the same architectures were also examined for the ANN and still the single hidden layer, single hidden node with a logistic activation function network proved superior to the others. In terms of RMSE, KNN was also better for the defender function model with $k=7$ identified as the value resulting in the minimum RMSE. Figure 10illustrates the RSME achieved by different values for $\mathrm{k}$ for the defender function.

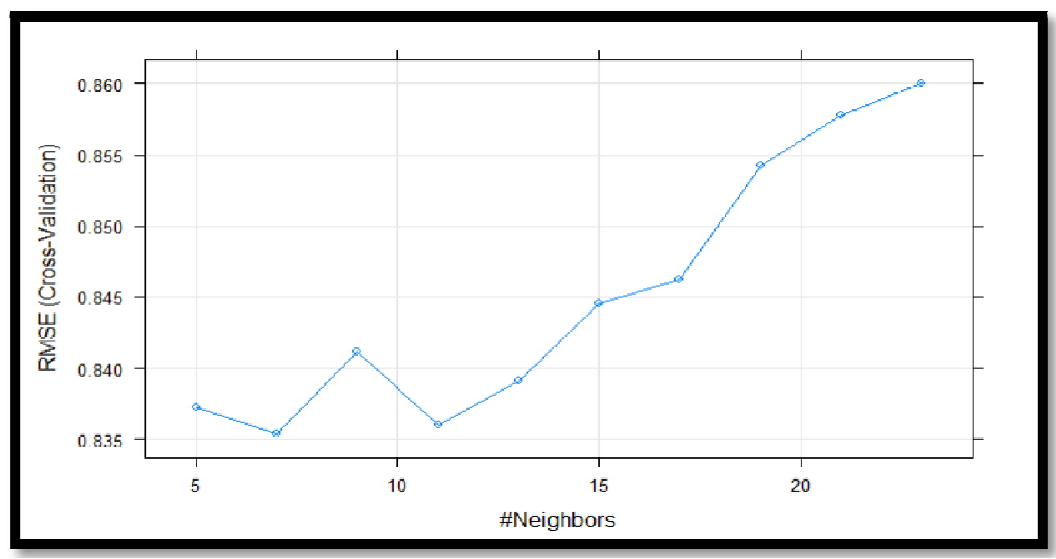

Figure 10: RMSE by Different K Values for the Defender Function

For the defender function market value prediction models, the Lasso instance was found to have the best predictive power in terms of both r-squared and the resulting RMSE.

\subsection{Midfielder Function Models}

In the same manner, market value prediction models for players in the midfielder function we implemented on the available performance and price data. Amongst the family of linear models, OLS, Ridge, Lasso and PCR were tested and assessed in terms of their explanatory power according to their $r$-squared and RMSE values. Lasso yielded the best results across indicators followed by, Ridge whose results were pretty close, then OLS and PCR. The results of the last 2 were quite far away in terms of the effectiveness measures.

On the non-linear model's side, ANN and KNN were implemented and examined. The attempted model architectures for the ANN were similar to the ones applied for the previous functions and the same conclusion was reached on both the best architecture and activation function sides. As for the KNN, $\mathrm{k}=9$ resulted in the minimum RMSE.

The change in RMSE with different values for $\mathrm{k}$ is demonstrated in figure 11. Still KNN performed better on all measures when compared to ANN in predicting the associated market value of players in the midfielder function.

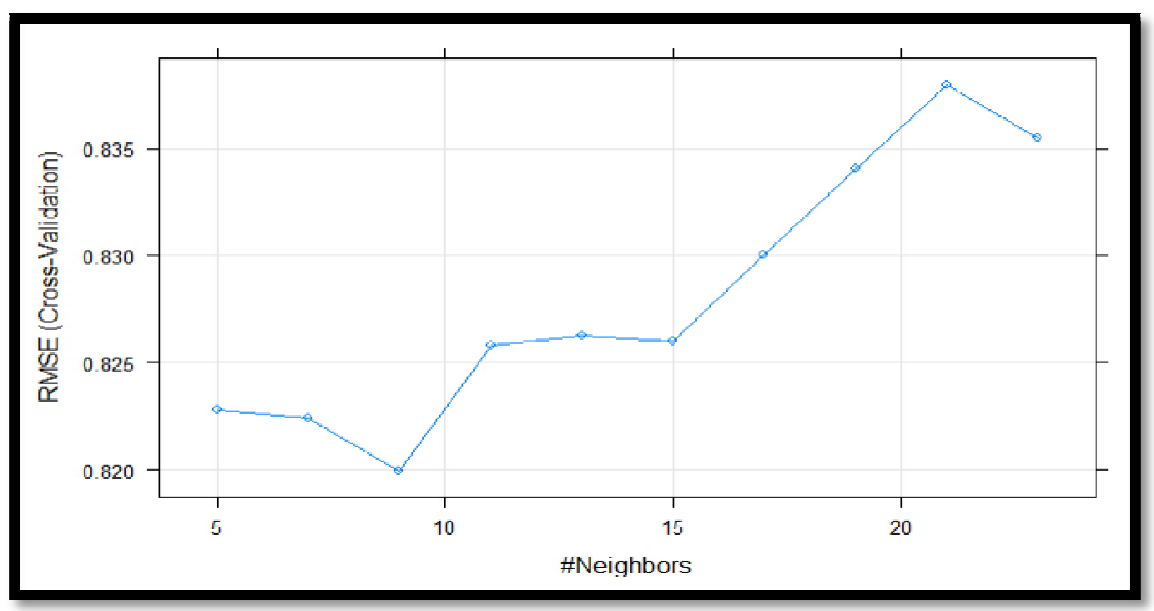

Figure 11: RMSE by Different K Values for the Midfielder Function

Similar to the other three functions, Lasso outperformed both the linear and non-linear models developed in terms of either the r-squared and RMSE.

In light of the results generated for all model attempts for every on pitch function, whether the linear or nonlinear ones, Lasso has proven superior in terms of its effectiveness in addressing the research problem across all the examined model evaluation indicators.

This finding matches the outcome of the vast majority of previous researches addressing the same problem in other leagues. It can be attributed to the linear nature of the underlying relation consistently found across all leagues, and hence the football market. Moreover, the shrinkage and regularization of variables achieved by the penalization concept proved effective in restraining the high diversity of performance variables. 


\section{Conclusion and Future Work}

For any industry, domain or sport, real superstars are short in supply and high in demand. Therefore, marginal increases in talent can lead to an extraordinary increase in the associated value. Extending that to football, transferring players from one club to another at the right price becomes a very challenging task. Hence the quantification of a player's performance and identifying its relation to his market value plays a significant role in being able to estimate the right price of a player in a given season.

In this study, a fully data driven methodology was devised to predict the market value of football players in the EPL according to their history of performance on pitch in previous seasons. In order to optimize the model and in attempt to achieve the closest possible estimates to the real market value of players, the approach was built in 2 phases. First, a segmentation model was developed to classify players according to their quantified performance into one of 3 segments: elite, high-potential or mainstream, where best performers are segmented as elite and worst performers as mainstream. Then several model attempts, both linear and non-linear, were applied to predict the expected equivalent market value of players according to their position, hence function, on pitch and history of performance by segment. Each model was tested for effectiveness and accuracy of prediction according to their r-squared and RMSE values. The linear pool of models included OLS, Ridge, Lasso and PCR whereas the non-linear included ANN and KNN. The Lasso model was found to be superior to the others for most of the functions, and was ranked second at its worst for only one function. Therefore, it can safely be selected as the champion model in this study this with the highest explanatory power and best accuracy level. ANN and KNN were modest in their capability to provide an accurate estimate of the response variable. Generally, all linear models always achieved better results than non-linear for all functions, hence are recommended for use in future studies of the same type.

Despite the fact that many researchers have recently addressed the same problem, none of them was implemented using EPL data or even tested for reliability in the Egyptian football market. Furthermore, the proposed approach is completely novel in structure in more than one way. A segmentation of players is done prior to the market value prediction in order to optimize the model performance. This step improved the prediction performance by more than $20 \%$ Additionally, a different model is fitted to each of the 4 distinctive on pitch functions to ensure assigning the right weights to the different skills needed according to the player's position.

Validation and testing of the proposed framework showed a decent prediction capability and promising results. However, it can possibly be extended in various ways, some of which are summarized below:

- Testing for effectiveness in a real business deal

- Scaling up the methodology to be applied on other leagues in different countries

- Examining the impact of including a popularity factor to compensate for the popularity of a player upon availability of related data

\section{References}

i. Eschweiler, M. \& Vieth, M. (2004). Preisdeterminanten bei Spieler transfers in der Fußball-Bundesliga, Zeitschrift für Betriebswirtschaft, 64,671-692.

ii. Hübl, L. and D. Swieter (2002). Der Spielemarkt in der Fußball-Bundesliga, Zeitschrift für Betriebswirtschaft. Ergänzungsheft, 72, 105-123.

iii. Garcia-del-Barrio, P. \& Pujol, F. (2004). Pay and Performance in the Spanish Soccer League: Who Gets the Expected Monopsony Rents?. Universidad de Navarra, 5,04.

iv. Battle, R., Bull, A., Hawkins, M., Hearne, S., Parkes, R. \& Thorpe, A. (2011). The untouchables, Football Money League. Manchester: Deloitte Sports Business Group, 2-40.

v. Carmichael, F., Forrest, D. \& Simmons, R. (1999). The Labour Market in Association Football: Who Gets Transferred and for How Much?. Bulletin of Economic Research, 51, 125-50.

vi. Lucifora, C. \& R. Simmons (2003). Superstar Effects in Sport: Evidence From Italian Soccer, Journal of Sports Economics, 4, 35-55.

vii. Tunaru, R. \& Clark, E. \& Viney, H., (2005). An option pricing framework for valuation of football players. Review of Financial Economics, Elsevier,14, 281-295.

viii. He, Y. (2015). Predicting Market Value of Soccer Players Using Linear Modeling Techniques. Technical Report. University of California, Berkeley.

ix. Ruijg, J., \& van Ophem, H. (2015). Determinants of football transfers. Applied Economics Letters, 22, 12-19.

x. Arndt, C. \& Brefeld, U. (2016). Predicting the future performance of soccer players. Statistical Analysis and Data Mining: The ASA Data Science Journal, 9, 373-382.

xi. He, M., Cachucho, R. \& Knobbe, A. (2015). Football Player's Performance and Market Value. Proceedings of the 2ndWorkshop on Machine Learning and Data Mining for Sports Analytics. CEUR Workshop Proceedings, 1970, 87-95.

xii. Wood, N.V. (1995). Perspectives on argument. Englewood Cliffs, NJ: Prentice Hall. Van den Berg, E.W.A. (2011). The Valuation of Human Capital in the Football Player Transfer Market. Erasmus University.

xiii. MacQueen, J. (1967). Some Methods for Classification and Analysis of Multivariate Observations. Proceedings of the 5th Berkeley Symposium on Mathematical Statistics and Probability, 1, 281-297.

xiv. James, G., Witten, D., Hastie, T. \& Tibshirani, R. (2014). An Introduction to Statistical Learning. Springer.

xv. Bruce, P. \& Bruce, A. (2017). Practical Statistics for Data Scientists. O’Reilly Media. 